\title{
Experimental Observation on the Retransmission of Radiation Side Effects
}

\author{
Yao Ruoyu ${ }^{1}$, Zhan $\mathrm{Hao}^{2}$, Zhang Xuguang ${ }^{1,2, ~ * ~}$ \\ ${ }^{1}$ Graduate School of Xuzhou Medical University, Xuzhou, China \\ ${ }^{2}$ Xuzhou Tumor Hospital, Xuzhou, China
}

Email address:

391589581@qq.com (Yao Ruoyu),1569033192@qq.com (Zhan Hao), zxgjh1992@qq.com (Zhang Xuguang)

*Corresponding author

\section{To cite this article:}

Yao Ruoyu, Zhan Hao, Zhang Xuguang. Experimental Observation on the Retransmission of Radiation Side Effects. Biochemistry and Molecular Biology. Vol. 4, No. 5, 2019, pp. 67-73. doi: 10.11648/j.bmb.20190405.11

Received: October 8, 2019; Accepted: November 8, 2019; Published: November 15, 2019

\begin{abstract}
Objective] To observe the retransmission of radiation side effects between cells. [Materials and Methods] The mouse ovarian cancer cell line NUTU19 was irradiated with 6MV-X-rays, and the culture medium was prepared for the first-generation conditioned medium. The first-generation effector cells were used to detect the NUTU19 cell line and the intestinal mucosal epithelial IEC- 6 cell line. The secretion of effector cells was a second-generation conditioned medium, and the second-generation effector cells NUTU19, IEC-6, and mouse lymphocytes were treated to measure cell viability and apoptosis. [Results] After treated with NUTU19 second-generation medium for 48 hours, the apoptosis rate of IEC-6 and NUTU19 cells was promoted $(p>0.05)$, and the apoptosis rate of lymphocytes was decreased $(p<0.05)$. After treatment with NUTU19 second-generation medium for $72 \mathrm{~h}$, the apoptosis of NUTU19 and IEC-6 was promoted ( $\mathrm{p}<0.05)$, and there was no effect on the apoptosis rate of lymphocytes ( $>0.05$ ). After treatment with IEC- 6 second-generation medium for $48 \mathrm{~h}$, the apoptosis of NUTU19 and IEC-6 was promoted $(p<0.05)$, and the apoptosis of lymphocytes was decreased $(p<0.05)$. After treated with IEC-6 second-generation medium for $72 \mathrm{~h}$, there was no effect on IEC- 6 and lymphocyte apoptosis $(\mathrm{p}>0.05)$. It promoted the apoptosis of NUTU19 $(\mathrm{p}<0.05)$. [Conclusion]: Under certain conditions, tumor cells, intestinal epithelial cells and lymphocytes can retransmit the damage of the side effect of radiation.
\end{abstract}

Keywords: Radiation Side Effect, Retransmission, Tumor Cells, Intestinal Epithelial Cells, Lymphocytes

\section{Introduction}

Since the first observation of the radiation side effect in 1992 [1], it has been found that the side effect in vivo is a "double-edged sword". For example, in patients with kidney cancer and breast cancer who are treated with local radiation, it is found that unaffected tumor tissue also has lesional damage [2-3]. A similar phenomenon was observed in animal experiments [4]. Experimental and clinical observations of more normal tissue damage in non-irradiated areas [5-10] can be over long distances and extra long time. When formulating the physical plan of radiotherapy, the side effect damage did not receive enough attention, and there was not enough theoretical support for its mechanism and how to antagonize it. Will this injury become one of the limiting factors in clinical radiotherapy?
Currently, we envision that the systemic response to local radiotherapy is long-distance and will last for a considerable amount of time. So, is there a side effect effector cell that retransmits the side effect? Just like a bacterial inflammatory reaction, a series of cells continue to advance the side effect? Which cells are involved in this process? To this end, we have designed the following experimental ideas. The ovarian cancer cell line NUTU19, which was grown by $6 \mathrm{MV}-\mathrm{X}$ line irradiation index, was prepared from the culture medium of the irradiated mouse ovarian cancer cell line, and the conditioned medium was treated with one generation of conditioned medium (NUTU19, rat small intestine crypt). The epithelial cell line IEC-6, SD rat blood lymphocytes) caused the effector cells to have a radiation side effect. Then, the normal culture solution is used to replace the first-generation conditioned medium, and the substance secreted by the effector cells is released into the new common culture solution, 
and then the culture solution is extracted to prepare the second-generation conditioned medium. Effector cells (NUTU19, IEC-6, murine lymphocytes) were treated with a second-generation conditioned medium to observe whether the second-generation conditioned medium had a side effect of deriving radiation. The specific experimental report is as follows.

\section{Materials and Methods}

\subsection{Experimental Animals and Experimental Cells}

SD rats, male, weighing $200 \pm 20 \mathrm{~g}$. They are all provided by the Animal Center of Xuzhou Medical University. rat ovarian cancer cell line NUTU19 (supplied by Shanghai Saisheng Co., Ltd.), rat small intestine crypt epithelial cell line IEC-6 (supplied by Shanghai Saisheng Co., Ltd.), SD rat blood lymphocytes. Conventional 1640 cell culture reagents and rat peripheral blood lymphocyte isolation reagents and techniques. Merck Biocck-8 kit and conventional techniques. Apoptosis was detected by Annexin V-FITC/PI double staining flow cytometer (model: FACSCalibur, manufacturer: BD). Real-time PCR instrument (model: iQ5, manufacturer: Bio-Rad), qRT-PCR technology to detect non-coding RNA expression. Varian UNIQUE accelerator, 6MV X-ray source pitch.

\subsection{Cell Culture Techniques and Conventional (Unconditional) Cultures}

NUTU19, IEC-6 cell line, rat lymphocytes, all using conventional 1640 medium, containing $10 \%$ fetal bovine serum (Hyclone), containing $100 \mathrm{U} / \mathrm{mL}$ penicillin and $100 \mathrm{U}$ $/ \mathrm{mL}$ streptomycin in MEM medium, The cultivation was carried out in a constant temperature incubator of $37.0 \mathrm{C}$ and $5 \%$ $\mathrm{CO} 2$. Using conventional methods for isolating rat peripheral blood lymphocytes.

\subsection{Conditioned Culture Solution Preparation}

\subsubsection{Mouse Source NUTU19 Cell Strain Generation Conditioned Medium Preparation}

Inoculate NUTU19 cell suspension (zero generation) in 24-well culture plates (40,000 cells/well), culture in a $37^{\circ} \mathrm{C} 5 \%$ CO 2 incubator, and photograph the cells before irradiation. Irradiation was performed with $6 \mathrm{MV}-\mathrm{X}$ rays at an air amount of 0 Gy and 2 Gy, respectively. Irradiated cells were cultured in a $37^{\circ} \mathrm{C}$ incubator for $24 \mathrm{~h}, 48 \mathrm{~h}$, and then photographed. The culture medium irradiated with $2 \mathrm{~Gy}$ was a first-generation conditioned medium, and the 0Gy irradiated was a control medium.

\subsubsection{Preparation of Second-Generation Conditioned Medium for Mouse NUTU19 and IEC-6 Cell Lines}

NUTU19 cell line (first generation effector cell) and IEC-6 cell line (first generation effector cell) were cultured for $48 \mathrm{~h}$ with NUTU19 generation conditioned medium, respectively. Replace fresh 1640 culture solution (ordinary unconditioned medium), and continue to culture the supernatant cells of the first generation of effector cells for 48 hours as NUTU19 second-generation conditioned medium and IEC-6 second-generation conditioned medium. The processing procedure is the same as above. Rat lymphocytes were unable to induce side effects, and the second-generation conditioned medium of mouse lymphocytes was not prepared. Second-generation conditioned medium contains no (or rarely contains) irradiated material compared to the first-generation conditioned medium. The second-generation conditioned medium contains substances secreted by the effector cells of the first-generation conditioned medium (first-generation effector cells) compared with the ordinary unconditioned broth.

\subsection{Side Effect Damage Experiment}

\subsubsection{Rat NUTU19 Cell Line Conditioned Medium for the First-Generation Effector Cells NUTU19, IEC-6, Rat Lymphocyte Injury Experiments}

The first generation of effector cells were treated with NUTU19 first-generation culture medium and cultured for $24 \mathrm{~h}$ and $48 \mathrm{~h}$ respectively. Using a normal unconditioned medium as a control, three parallel samples were set. The cck8 technique detects cell viability. Apoptosis levels were detected by Annexin V-FITC/PI double staining flow cytometry.

\subsubsection{Rat Second-Generation Conditioned Medium Damage Test}

The second-generation effector cells NUTU19, IEC-6, and mouse lymphocytes were treated with NUTU19 second-generation medium and IEC-6 second-generation conditioned medium, respectively, for $48 \mathrm{~h}$ and $72 \mathrm{~h}$. Ordinary unconditioned medium was used as a control. Set up 3 parallel samples.

CCK8 technique to detect cell viability. After $48 \mathrm{~h}$ and $72 \mathrm{~h}$ of culture, $10 \mathrm{ul}$ of CCK- 8 was added to each well. After mixing, NUTU18 and IEC-6 cells were incubated for $2 \mathrm{~h}$, and lymphocytes were incubated for $6 \mathrm{~h}$. The absorbance at $450 \mathrm{~nm}$ was measured. The microplate reader reads the OD value of the sample to be tested and the blank control at $450 \mathrm{~nm}$, and records the OD value of each sample to be measured. The OD value of the blank control is recorded as a blank value, and the final value $=$ measured value - blank value. Apoptosis levels were detected by Annexin V-FITC/PI double staining flow cytometry.

\subsection{Statistical Analysis}

Data entry uses excel, logical proofreading, and generates a database. The experimental data were expressed as $\mathrm{x} \pm \mathrm{s}$, and the data was analyzed using SPSS 19.0 statistical software. Two independent samples t-test were used for comparison between the two groups. $\mathrm{P}<0.05$ was considered statistically significant.

\section{Result and Discussion}

\subsection{Mouse Epithelial Cell Fraction}

CCK8 experimental part 
Table 1. Detection of cck8 in NUTU19 cell line by $6 M V-X$-ray irradiation.

\begin{tabular}{lll}
\hline Group & $\mathbf{2 4 h}$ & $\mathbf{4 8 h}$ \\
\hline $0 \mathrm{~Gy}$ & $0.714 \pm 0.013$ & $1.178 \pm 0.011$ \\
$2 \mathrm{G}$ & $0.561 \pm 0.008$ & $0.664 \pm 0.021$ \\
$t$ & 17.361 & 37.554 \\
$p$ & $<0.001$ & $<0.001$ \\
\hline
\end{tabular}

Irradiation has a significant inhibitory effect on the proliferation of tumor cells.

NUTU19 Tumor cell lines (NUTU19), normal epithelial cells (IEC-6), and lymphocytes treated with the primary culture solution were cultured for $24 \mathrm{~h}$ and $48 \mathrm{~h}$, respectively, and parallel controls were set.

Table 2. (IEC-6) and lymphocytes treated with NUTU19 generation conditioned medium.

\begin{tabular}{lll}
\hline Group & $\mathbf{2 4 h}$ & $\mathbf{4 8 h}$ \\
\hline NUTU19 Control group & $0.316 \pm 0.014$ & $1.099 \pm 0.010$ \\
NUTU19 & $0.208 \pm 0.024$ & $0.377 \pm 0.004$ \\
$t$ & 6.732 & 116.110 \\
$p$ & 0.003 & $<0.001$ \\
IEC-6 Control group & $0.186 \pm 0.011$ & $0.532 \pm 0.023$ \\
IEC-6 & $0.152 \pm 0.012$ & $0.058 \pm 0.002$ \\
$t$ & 3.618 & 35.561 \\
$p$ & 0.002 & $<0.001$ \\
Lymphocyte control group & $0.152 \pm 0.009$ & $0.158 \pm 0.008$ \\
Lymphocyte & $0.145 \pm 0.013$ & $0.152 \pm 0.004$ \\
$t$ & 0.767 & 1.162 \\
$p$ & 0.486 & 0.310 \\
\hline
\end{tabular}

Compared with the control group, proliferation of NUTU19 cells and IEC-6 cells was inhibited, and there was no significant effect on lymphocyte proliferation activity.
OD results of ckk8 detection of effector cells in the second-generation culture medium.

Table 3. NUTU19 second-generation conditioned medium treatment NUTU19, IEC-6, lymphocyte cck8 detection OD results.

\begin{tabular}{lll}
\hline & $\mathbf{4 8 h}$ & $\mathbf{7 2 h}$ \\
\hline NUTU19 & & $3.340 \pm 0.017$ \\
control group & $2.547 \pm 0.003$ & $0.626 \pm 0.024$ \\
Second generation processing group & $0.500 \pm 0.009$ & 90.356 \\
$t$ & 194.67 & $<0.001$ \\
$p$ & $<0.001$ & $2.756 \pm 0.060$ \\
IEC-6 & & $0.209 \pm 0.009$ \\
control group & $0.920 \pm 0.024$ & 41.859 \\
Second generation processing group & $0.041 \pm 0.010$ & $<0.001$ \\
$t$ & 33.373 & \\
$p$ & $<0.001$ & $0.334 \pm 0.048$ \\
Lymphocyte & & $0.349 \pm 0.017$ \\
Lymphocyte control group & $0.338 \pm 0.006$ & 0.291 \\
Second generation processing group & $0.331 \pm 0.038$ & 0.785 \\
\hline
\end{tabular}

NUTU19 second-generation cultured NUTU19 cells and IEC-6 cells for 48h, 72h, compared with the control group, the relative survival rate of cells decreased. Lymphocytes were treated for $48 \mathrm{~h}$ and $72 \mathrm{~h}$, and there was no significant effect on the relative survival rate of cells compared with the control group.

Table 4. IEC results for CK-6 second-generation culture treatment of NUTU19, IEC-6, and lymphocyte cck8.

\begin{tabular}{lll}
\hline & $\mathbf{4 8 h}$ & $\mathbf{7 2 h}$ \\
\hline NUTU19 & & $3.340 \pm 0.017$ \\
control group & $2.547 \pm 0.003$ & $3.318 \pm 0.054$ \\
IEC-6 second generation culture solution treatment & $2.854 \pm 0.070$ & 0.387 \\
$t$ & 4.435 & 0.718 \\
$p$ & 0.011 & $2.756 \pm 0.060$ \\
IEC-6 & & $2.552 \pm 0.034$ \\
control group & $0.920 \pm 0.024$ & 2.954 \\
IEC-6 second generation culture solution treatment & $0.864 \pm 0.039$ & 0.042 \\
$t$ & 1.221 & $0.334 \pm 0.048$ \\
$p$ & 0.289 & $0.359 \pm 0.002$ \\
Lymphocyte & & 0.504 \\
Lymphocyte control group & $0.338 \pm 0.006$ & 0.640 \\
$t$ & $0.336 \pm 0.038$ & \\
\hline
\end{tabular}


The NUTC19 cell line was cultured in the second generation of IEC-6, and the 48-hour survival rate was slightly increased, and the 72-hour survival rate was restored. Treatment of IEC-6 cell lines showed no change in 48-hour survival rate and a decrease in 72-hour survival rate. Treatment of lymphocytes for $48 \mathrm{~h}, 72 \mathrm{~h}$ had no effect on cell viability.

The second-generation conditioned medium can reduce the proliferation activity of the cells, indicating that the proliferation damage of the side effect can be transmitted, and the intensity of the transmitted damage is also significant. Little influence on lymphocytes. Different cell secretions and different effector cells have different delivery effects.

\subsection{Apoptotic Part}

\subsubsection{Treatment of Mouse-Derived Cells with a Generation of NUTU19 Conditioned Medium}

24 hour result

Table 5. Apoptosis rate (\%) of NUTU19, IEC-6, and lymphocytes after treatment of cells with primary culture for $24 \mathrm{~h}$ and $48 \mathrm{~h}$.

\begin{tabular}{llll}
\hline & IEC-6 & NUTU19 & Lymphocyte \\
\hline $24 \mathrm{~h}$ & & & $1.74 \pm 0.11$ \\
control group & $2.47 \pm 0.12$ & $7.59 \pm 0.64$ & $0.99 \pm 0.09$ \\
First generation culture fluid group & $3.62 \pm 0.39$ & $1.20 \pm 2.08$ & 4.948 \\
$t$ & 2.831 & 2.850 & 0.001 \\
$p$ & 0.047 & 0.046 & Lymphocyte \\
$48 \mathrm{~h}$ & IEC-6 & NUTU19 & $1.56 \pm 0.22$ \\
control group & $2.67 \pm 0.12$ & $5.75 \pm 0.20$ & $1.09 \pm 0.23$ \\
First generation culture fluid group & $37.66 \pm 0.75$ & $23.10 \pm 2.12$ & 1.481 \\
$t$ & 46.354 & 8.145 & 0.213 \\
\hline
\end{tabular}

After treated with the culture medium for 24 hours, the apoptosis rate of IEC-6 increased, the apoptosis rate of NUTU19 decreased, and the apoptosis rate of lymphocytes decreased. After treatment with a culture medium for 48 hours, the apoptosis of IEC-6 and NUTU19 was significantly promoted, and the apoptosis rate of lymphocytes was not affected. The 48-hour result was more stable. The treatment time of the second generation culture solution was extended to 48 hours.

\subsubsection{Conditioned Culture Solution Treatment of Mouse-Derived Cells Results}

48-hour result

NUTU19 second generation culture solution:

Table 6. Apoptosis rate (\%) of NUTU19 cell line, normal epithelial cells (IEC-6) and lymphocytes after treatment with NUTU19 second-generation medium for $48 h$ and $72 h$.

\begin{tabular}{|c|c|c|c|}
\hline & IEC-6 & NUTU19 & Lymphocyte \\
\hline \multicolumn{4}{|l|}{$48 \mathrm{~h}$} \\
\hline control group & $4.23 \pm 0.08$ & $1.49 \pm 0.05$ & $1.39 \pm 0.23$ \\
\hline NUTU19 second generation culture fluid group & $6.27 \pm 0.23$ & $11.18 \pm 1.36$ & $0.33 \pm 0.05$ \\
\hline$t$ & 8.402 & 7.138 & 4.497 \\
\hline$p$ & 0.001 & 0.002 & 0.010 \\
\hline $72 \mathrm{~h}$ & IEC-6 & NUTU19 & Lymphocyte \\
\hline control group & $3.92 \pm 0.17$ & $1.49 \pm 0.12$ & $0.57 \pm 0.23$ \\
\hline$t$ & 21.92 & 25.503 & 1.372 \\
\hline$p$ & $<0.001$ & $<0.001$ & 0.242 \\
\hline
\end{tabular}

After treated with NUTU19 second-generation medium for 48 hours, the apoptosis rate of IEC- 6 and NUTU19 cells was promoted, and the apoptosis rate of lymphocytes was reduced. After treated with NUTU19 second-generation medium for 72 hours, it promoted the apoptosis of NUTU19 and IEC-6, and had no effect on the apoptosis rate of lymphocytes.

IEC-6 second generation culture solution:

Table 7. Apoptosis rate (\%) of NUTU19, IEC-6, and lymphocytes after treatment with IEC-6 second-generation medium for $48 \mathrm{~h}$ and $72 \mathrm{~h}$.

\begin{tabular}{llll}
\hline & IEC-6 & NUTU19 & Lymphocyte \\
\hline $48 \mathrm{~h}$ & & & \\
control group & $1.77 \pm 0.05$ & $1.05 \pm 0.08$ & $1.39 \pm 0.23$ \\
IEC-6 second generation culture fluid group & $2.88 \pm 0.27$ & $2.08 \pm 0.02$ & $0.70 \pm 0.06$ \\
$t$ & 3.978 & 12.59 & 2.867 \\
$p$ & 0.016 & $<0.001$ & Lymphocyte \\
$72 \mathrm{~h}$ & IEC-6 & NUTU19 & $0.047 \pm 0.23$ \\
control group & $3.71 \pm 0.11$ & $0.42 \pm 0.07$ & $0.55 \pm 0.14$ \\
IEC-6 second generation culture fluid group & $5.02 \pm 0.52$ & $2.45 \pm 0.27$ \\
$t$ & 2.487 & 7.314 & 0.08 \\
$p$ & 0.068 & 0.001 & 0.469 \\
\hline
\end{tabular}


After treated with IEC-6 second-generation medium for 48 hours, it promoted the apoptosis of NUTU19 and IEC-6, and decreased the apoptosis of lymphocytes. After treated with IEC-6 second-generation medium for $72 \mathrm{~h}$, there was no effect on the apoptosis of IEC- 6 and lymphocytes. It promotes the apoptosis of NUTU19.

The second-generation culture medium promoted apoptosis to IEC-6 and NUTU19, and seemed to promote the promotion of NUTU19. There is no effect on lymphocytes to promote apoptosis. It is indicated that the apoptotic expression of the side effect can be re-transferred through the secretion of effector cells, and the transmission effect on tumor cells and epithelial cells is stronger.

Lymphocytes are not sensitive to the primary and secondary conditioned medium from which epithelial cells are derived.

In clinical radiotherapy, late radiation therapy can be present for a long time outside the irradiation area. Is there a side effect space transfer and time transfer?

$\mathrm{J}$ Dong $\mathrm{C}$ et al [11] irradiated human small cell lung cancer cell line H446, which induced U937 macrophages to produce secondary side effects on human bronchial epithelial cells BEAS-2B not directly co-cultured with target cells, and target cells produced cytokines. The side effects can be transmitted by macrophages. When the target cell is a lung fibroblast [12], the macrophage also has a function of transmitting a side effect signal. Lung cancer cells, bronchial epithelial cells, and lung fibroblasts are all fixed or attached to tissues, and macrophages are migrating. It can be speculated that macrophages can act as "messengers" in addition to their biological characteristics, "movable" is also an important factor. Then, in addition to transmitting the signal, does the messenger have a corresponding change? Do other cells have a similar effect?

Our previous work [13] and current work, observed from the level of cell proliferation and apoptosis, radiation side effects can be transmitted between cells. Tumor cells, epithelial cells, and lymphocytes are both side effector cells and delivery cells, and the second-generation side effects transmitted by them are quite powerful. Show that they are active participation, not just a "messenger." It can be understood that they respond to side effects at the cellular level.

Different from the heavy ion micro-irradiation, using the clinical irradiation method, this experiment observed that the tumor cells produced higher-efficiency first- and second-generation conditioned medium, and the side-effect response intensity of the tumor cells to the culture medium was also higher. This is consistent with the observation that distant tumors are inhibited by radiotherapy in the clinic. Other investigators [14-15] also used conditioned cells to prepare conditioned medium, and tumor cell damage was also observed with normal epithelial damage. In this experiment, intestinal epithelial cells can act as side effector cells, delivery cells, and re-effector cells. The digestive function of the patient during radiotherapy decline may be partly related to this.
Normal tissue side effects damage other than the intestinal epithelium has also been observed. Corresponding lesions were found in brain tissue, lung tissue, hair follicles, spleen, testis, etc. outside the target area $[9,16,19,20]$. Responses outside the target area $[5,17]$ were associated with changes in immune function, repair of DNA damage, and even persisted 7 months after irradiation [19]. Can their side effects last for so long, is it a simple first-order side effect? Therefore, we speculate the existence of side effect transmission.

There is not much research data on lymphocytes affected by side effects. Sun Daqing et al. [21] irradiated human lymphoblasts (AHH-1) with 60 Co $\gamma$-rays, and found that the radiation side effect has a large number of signaling molecules transport and protein transport. Other studies have looked at lymphocytes from the perspective of the body's immune response after radiotherapy. It is believed that after tumor radiotherapy, a large number of cytokines, such as NF-KB, high mobility group protein 1 , etc., will promote the maturation of dendritic cells (DC) and also promote DC to draining lymph nodes. Transfer [22-26]. When the DC migrates to the lymph node, the DC surface protein interacts with the $\mathrm{T}$ cell surface protein to activate the $\mathrm{T}$ cell. The final effector $\mathrm{T}$ cells leave the lymph nodes and reach the irradiated and unirradiated tumor tissues. These antigen-recognizing $\mathrm{T}$ cells act on the tumor at the site where they occur, causing regression of the distal tumor. In these studies, lymphocytes were viewed as a passive link.

In this study, the use of tumor cell lines and intestinal epithelial cell lines failed to induce lymphocyte death and apoptosis effects. May be related to radiation dose, culture time, single cell and other factors. The underlying mechanisms of the bystander effect are multifaceted, depending on biological factors (irradiated cells, bystander cells, characteristics of the intercellular environment) and physical factors (radiation dose, rate and type, time). We believe that the mechanisms of side effects and side effects are complex and have many unknowns at the cellular, tissue, systemic, and global levels. The existing research accumulation is not enough to propose an overall correct path. In general, under certain conditions, tumor cells, normal epithelial cells, and lymphocytes are able to transmit radiation side effects. So, what is the significance of side effects that are transmitted between cells, between tissues, between systems, and at the overall level?

\section{Conclusion}

Based on microscopic illumination and cytology studies, the definition of radiation side effects [27] is that when cells or tissues are irradiated, some of the irradiated cells transmit signals to surrounding cells, and the corresponding non-target cells receive signals and generate corresponding signals. Biological effects. It is a point-to-point relationship between irradiated cells and side effector cells. Recognizing the phenomenon of side effect transmission, we can study the side effects at the organization, organ, system and overall level. It 
is possible that the side effect is not a point-to-point effect, but a multi-point linear or even a network-like relationship, and even There is a role of time dimension. Tumor regression, normal tissue inflammation, organ fibrosis, secondary tumors, etc. after live irradiation are the result of this network shock, gene mutation, genetic instability, DNA damage, chromosome breakage and mutation, cell proliferation and apoptosis., inflammatory response, tumorigenic transformation, etc is a wave of network shocks. [28]. With the development of the mechanism of radiation injury, it can not only guide the treatment of tumor, but also play an important role in the protection of radiation injury. At present, the retransmission of side effect is only limited to the basic theoretical research. How to use this effect to increase the radiotherapy effect and reduce the radiation damage remains to be further studied. With the deepening of the understanding of the transmission of the side effect, we will further expand the connotation and extension of the traditional radiation side effect. Based on the known characteristics of bystander effect, it can be inferred that it has a significant and constructive effect on tumor radiotherapy: by using its effect on non irradiated cells, it can reduce the radiation dose, narrow the radiation range and achieve the same therapeutic effect; by blocking the transmission of its effect on adjacent normal tissues, it can protect normal tissues and improve the target specificity of radiotherapy The role of sex. It is believed that with the development of the research, the mechanism of radiation-induced bystander effect transfer will be more thoroughly understood, and finally it can be applied to the practical work of radiotherapy and protection.

\section{References}

[1] Correspondence Re: H. Nagasawa and J. B. Little, Induction of sister chromatid exchanges by extremely low doses of alpha-particles. Cancer Res., 52: 6394-6396, 1992. Cancer Res 1993; 53: 2188.

[2] Wersäll PJ, Blomgren $\mathrm{H}$, Pisa $\mathrm{P}$, et al. Regression of non-irradiated metastases after extracranial stereotactic radiotherapy in metastatic renal cell carcinoma. Acta Oncol 2006; 45: 493-497.

[3] Kulcenty K, Piotrowski I, Zaleska K, et al. Wound fluids collected postoperatively from patients with breast cancer induce epithelial to mesenchymal transition but intraoperative radiotherapy impairs this effect by activating the radiation-induced bystander effect. Sci Rep 2019; 9: 7891.

[4] Camphausen K, Moses MA, Ménard C, et al. Radiation abscopal antitumor effect is mediated through p53. Cancer Res 2003; 63: 1990-1993.

[5] Calveley VL, Jelveh S, Langan A, et al. Genistein can mitigate the effect of radiation on rat lung tissue. Radiat Res 2010; 173: 602-611.

[6] Diot Q, Kavanagh B, Schefter T, et al. Regional normal lung tissue density changes in patients treated with stereotactic body radiation therapy for lung tumors. Int J Radiat Oncol Biol Phys 2012; 84: 1024-1030.
[7] Chang JY, Zhang X, Wang X, et al. Significant reduction of normal tissue dose by proton radiotherapy compared with three-dimensional conformal or intensity-modulated radiation therapy in Stage I or Stage III non-small-cell lung cancer. Int J Radiat Oncol Biol Phys 2006; 65: 1087-1096.

[8] Desai S, Kobayashi A, Konishi T, et al. Damaging and protective bystander cross-talk between human lung cancer and normal cells after proton microbeam irradiation. Mutat Res 2014; 763-764: 39-44.

[9] Siva S, Lobachevsky P, MacManus MP, et al. Radiotherapy for Non-Small Cell Lung Cancer Induces DNA Damage Response in Both Irradiated and Out-of-field Normal Tissues. Clin Cancer Res 2016; 22: 4817-4826.

[10] Feiock C, Yagi M, Maidman A, et al. Central Nervous System Injury - A Newly Observed Bystander Effect of Radiation. PLoS One 2016; 11: e0163233.

[11] Dong $\mathrm{C}, \mathrm{He} \mathrm{M}, \mathrm{Tu} \mathrm{W}$, et al. The differential role of human macrophage in triggering secondary bystander effects after either gamma-ray or carbon beam irradiation. Cancer Lett 2015; 363: 92-100.

[12] Fu J, Yuan D, Xiao L, et al. The crosstalk between $\alpha$-irradiated Beas-2B cells and its bystander U937 cells through MAPK and NF- $\kappa B$ signaling pathways. Mutat Res 2016; 783: 1-8.

[13] Yang S, Xu J, Shao W, et al. Radiation-Induced Bystander Effects in A549 Cells Exposed to 6 MV X-rays. Cell Biochem Biophys 2015; 72: 877-882.

[14] Kong EY, Cheng SH. Induction of autophagy and interleukin 6 secretion in bystander cells: metabolic cooperation for radiation-induced rescue effect?J Radiat Res 2018; 59: 129-140.

[15] Bill MA, Srivastava K, Breen C, et al. Dual effects of radiation bystander signaling in urothelial cancer: purinergic-activation of apoptosis attenuates survival of urothelial cancer and normal urothelial cells. Oncotarget 2017; 8: 97331-97343.

[16] Khan MA, Hill RP. Partial volume rat lung irradiation: an evaluation of early DNA damage. Int J Radiat Oncol Biol Phys 1998; 40: 467-476.

[17] Koturbash I, Rugo RE, Hendricks CA, et al. Irradiation induces DNA damage and modulates epigenetic effectors in distant bystander tissue in vivo. Oncogene 2006; 25: 4267-4275.

[18] Koturbash I, Zemp FJ, Kutanzi K, et al. Sex-specific microRNAome deregulation in the shielded bystander spleen of cranially exposed mice. Cell Cycle 2008; 7: 1658-1667.

[19] Tamminga J, Koturbash I, Baker M, et al. Paternal cranial irradiation induces distant bystander DNA damage in the germline and leads to epigenetic alterations in the offspring. Cell Cycle 2008; 7: 1238-1245.

[20] Ilnytskyy Y, Koturbash I. Radiation-induced bystander effects in vivo are epigenetically regulated in a tissue-specific manner. Environ Mol Mutagen 2009; 50: 105-113.

[21] Sun DQ, Xiao Y, Han L, et al. High expression of AP1S1 in radiation side effect and its role in side effects. Radioprotection 2010, 30 (6): 356.

[22] Herrera FG, Bourhis J. Radiotherapy combination opportunities leveraging immunity for the next oncology practice. CA Cancer J Clin 2017; 67: 65-85. 
[23] Tang D, Kang R, Zeh HJ. High-mobility group box 1, oxidative stress, and disease. Antioxid Redox Signal 2011; 14: 1315-1335.

[24] Garg AD, Krysko DV, Verfaillie T, et al. A novel pathway combining calreticulin exposure and ATP secretion in immunogenic cancer cell death. EMBO J 2012; 31: 1062-1079.

[25] Ohshima Y, Tsukimoto M, Takenouchi T, et al. gamma-Irradiation induces $\mathrm{P} 2 \mathrm{X}$ (7) receptor-dependent ATP release from B16 melanoma cells. Biochim Biophys Acta 2010; 1800: $40-46$.
[26] Elliott MR, Chekeni FB, Trampont PC, et al. Nucleotides released by apoptotic cells act as a find-me signal to promote phagocytic clearance. Nature 2009; 461: 282-286.

[27] Mukherjee S. Radiation-induced bystander phenomenon: insight and implications in radiotherapy. Int J Radiat Biol 2019; 95: 243-263.

[28] Zhang X, Pan Y, Shao CL. Research progress on the bystander effect of radiotherapy in vivo. International Journal of Radiation Medicine and Nuclear Medicine 2017 (41) 3: 209-213. 